\title{
Is Additional Gastrectomy Required for Elderly Patients after Endoscopic Submucosal Dissection with Endoscopic Curability C-2 for Early Gastric Cancer?
}

\author{
Waku Hatta ${ }^{a}$ Takuji Gotoda $^{b}$ Tomoyuki Koike ${ }^{a}$ Kaname Uno ${ }^{a}$ Naoki Asano ${ }^{a}$ \\ Akira Imatani $^{a} \quad$ Atsushi Masamune $^{a}$ \\ aDivision of Gastroenterology, Tohoku University Graduate School of Medicine, Sendai, Japan; bivision of \\ Gastroenterology and Hepatology, Department of Medicine, Nihon University School of Medicine, Tokyo, Japan
}

\section{Keywords \\ Early gastric cancer $\cdot$ Endoscopic curability C-2 Elderly . \\ Endoscopic submucosal dissection}

\begin{abstract}
s
Background: With the ongoing growth of the aged population, the number of elderly patients suffering from gastric cancer has increased in Japan. Since the frequency of lymph node metastasis (LNM) in patients after endoscopic submucosal dissection (ESD) with endoscopic curability (eCura) C-2 for early gastric cancer (EGC) is relative low, the following question can be raised: "Is additional gastrectomy required for elderly patients with such criteria for ESD?" Summary: For therapeutic decision-making after ESD with eCura C-2, the risk of all-cause mortality and impaired quality of life (QoL) should thus be evaluated. Risk stratification of LNM and gastric cancer-specific mortality was established by the eCura system; however, it remains unclear how much these categories and treatment selection affect all-cause mortality. The contribution of prognostic tools for predicting all-cause mortality was noted to vary across the studies of patients with EGC; thus, further studies that investigate comprehensive geriatric assessment (CGA) may be required. Regarding the QoL, studies on elderly patients remain to be lacking.
\end{abstract}

Furthermore, one of the issues with CGA and QoL tools is that they are time consuming. Key Messages: Combined evaluation of risk stratification of gastric cancer-specific mortality by the eCura system and risk of nongastric cancerrelated mortality and impaired QoL may be the current optimal method to decide treatment strategy after ESD with eCura C-2 for EGC among elderly patients. A large-scale prospective study that investigates CGA domains is required to identify predictors of all-cause mortality and impaired QoL, and a more easily usable tool should be developed.

(c) 2021 S. Karger AG, Basel

\section{Introduction}

Although gastric cancer remains one of the major cancers worldwide, gastric cancer incidence is expected to follow the current downward trend due to the lower frequency of Helicobacter pylori infection in the younger generation and the increasing of aging population in Japan. In this process, elderly patients with gastric cancer will further increase [1]; thus, an appropriate management for elderly patients with gastric cancer is required to be developed. karger@karger.com

www.karger.com/dig

Karger ${ }^{\prime \prime}=$
(C) 2021 S. Karger AG, Basel

(2021 S. Karger AG, Basel
Correspondence to:

Takuji Gotoda, takujigotoda@yahoo.co.jp 


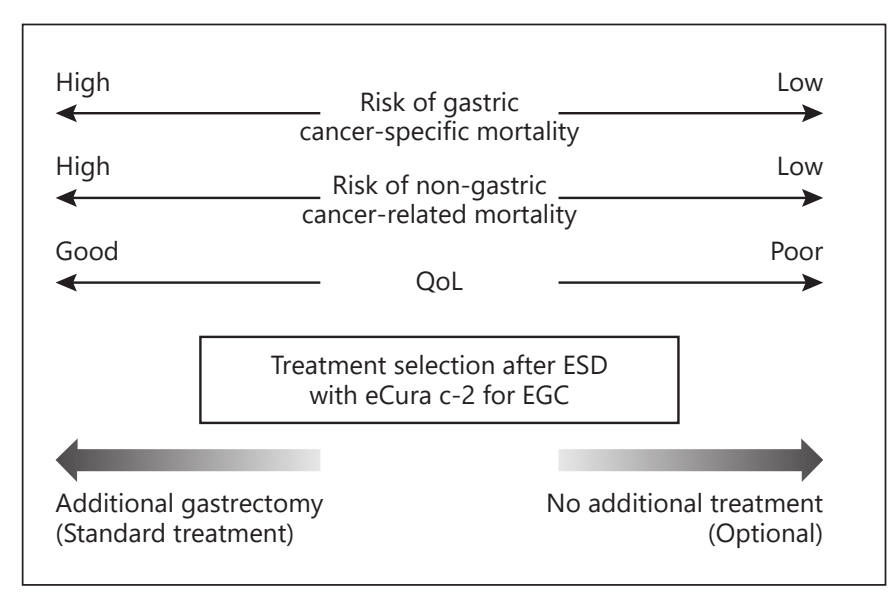

Fig. 1. Factors associated with therapeutic decision-making in elderly patients after ESD with eCura C-2 for EGC. ESD, endoscopic submucosal dissection; EGC, early gastric cancer; QoL, quality of life.

Endoscopic mucosal resection has now been widely accepted worldwide as a minimally invasive treatment for early gastric cancer (EGC) with negligible risk of lymph node metastasis (LNM). In Korea and Japan, endoscopic mucosal resection has already been replaced by endoscopic submucosal dissection (ESD), which is now a standard treatment method for EGC in these countries.

With the accumulation of evidence, the indication of endoscopic resection and the criteria for curative resection have been expanded [2]. When the lesion does not meet the criteria for curative resection, additional gastrectomy with lymphadenectomy is recommended as the standard of care $[3,4]$. However, the rate of LNM in such patients ranges from $5 \%$ to $10 \%$. Furthermore, some patients do not experience additional gastrectomy because of old age, comorbidity, patients' preference, and so on. As described above, the number of elderly patients with gastric cancer has been increasing in Japan. Thus, the following question can be raised: "Is additional gastrectomy required for elderly patients after ESD for EGC that does not meet the curative criteria"? In this review, we examine the current knowledge of such patients and discuss what is needed to overcome this issue.

\section{Aging of Gastric Cancer Patients}

Although the definition of "elderly" varies across the studies, institutions, and countries, the threshold age of $\geq 70$ or $\geq 75$ years is often used in politics [5]. The number of elderly patients with cancer has been increasing worldwide, and absolute numbers of new cancer cases in individuals aged 65 or above are predicted to double in 2035 with respect to the 2012 numbers [6].

Gastric cancer is particularly common in Eastern Asian countries. Japan is one of the front-runners among countries with a rapidly aging society, and the proportion of elderly patients among those with gastric cancer has been noted to be on the rise. Indeed, the peak age of patients with gastric cancer has increased from 60-64 years old in 1990 to $75-79$ years old in 2014. Thus, gastric cancer is now identified as a disease of the elderly population in Japan. The therapeutic decision in elderly patients can be complex compared to that in nonelderly patients because of a variety of comorbidity, physiologic function, and so on. For appropriate therapeutic decision-making in such patients, it is important to consider age-related physiological change and outcome as well as the cancerrelated outcome.

\section{Curability Criteria after ESD for EGC}

In the latest guidelines [4], the curability criteria after ESD for EGC are classified into endoscopic curability (eCura) A, B, and C-1/C-2, which corresponds to curative resection, expanded curative resection, and noncurative resection, respectively, in the guidelines of the previous version. When piecemeal resection or positive horizontal margin is the sole noncurative factor after ESD, the tumor is classified as eCura C-1. Meanwhile, all tumors that do not meet the criteria for eCura $\mathrm{A} / \mathrm{B} / \mathrm{C}-1$ are classified as eCura C-2.

\section{What Knowledge Is Needed for Therapeutic Decision-Making in Elderly Patients after ESD with eCura C-2 for EGC?}

Although additional gastrectomy is the standard treatment method for EGC with eCura C-2 after ESD, many elderly patients do not undergo this treatment in clinical practice. According to a multicenter study [7], the rate of undergoing additional gastrectomy differed with the different age groups: $70.0 \%, 54.7 \%$, and $20.0 \%$ in $<70,70-79$, and $\geq 80$-year-old patients, respectively. In deciding the treatment strategy after ESD with eCura C-2, the risk of nongastric cancer-related mortality as well as that of gastric cancer-specific mortality has to be taken into account [8] (Fig. 1). Furthermore, the International Society of Ge-
Hatta/Gotoda/Koike/Uno/Asano/ Imatani/Masamune 
riatric Oncology recently encouraged greater focus on the quality of life (QoL) of elderly people living with cancer [9]. Thus, gastric and nongastric cancer-related mortality and QoL should be considered in therapeutic decisionmaking in elderly patients with eCura C-2 after ESD for EGC (Fig. 1).

\section{Current Knowledge about ESD with eCura C-2 for EGC}

A number of previous studies have focused on longterm prognosis, LNM, and recurrence in patients with eCura C-2 after ESD for EGC. Thus, the current knowledge in this field is mainly about tumor-related outcome.

\section{Long-Term Prognosis}

Several studies have evaluated long-term prognosis in patients after ESD with eCura C-2 for EGC [8]. A largescale multicenter study showed favorable disease-specific survival (DSS) in both patients with additional gastrectomy and no additional treatment after ESD with eCura C-2 for EGC (98.8\% vs. 97.5\% in 5 years) [9], although additional gastrectomy was noted to decrease the risk of gastric cancer-specific mortality by one-third [10]. Furthermore, the DSS did not differ across hospital volumes [11]. Meanwhile, the 5-year overall survival (OS) rates in patients with additional gastrectomy and no additional treatment were $92.6 \%$ and $75.2 \%$, respectively [9], the difference of which is possibly due to selection bias. These rates decrease as the patients get older $(84.7 \%$ and $73.7 \%$, respectively, in 5 years in patients aged $\geq 80$ ) [7].

\section{The Prevalence of LNM and the Use of the eCura}

System for Predicting the Risk of LNM

A recent meta-analysis reported the prevalence of LNM (95\% confidence interval) as 8.0\% (7.1-8.9\%) in patients after ESD with eCura C-2 for EGC [12]. Furthermore, a scoring system consisting of 5 pathological factors for predicting the risk of LNM, called the eCura system, was established (Fig. 2) [13]. A mobile application of this model (English, Chinese, and Japanese versions) was also developed [14]. The eCura system has 3 risk categories with the LNM rates of $2.5 \%, 6.7 \%$, and $22.7 \%$ in the low-, intermediate-, and high-risk categories, respectively. The validity of the weight of each pathological factor in the eCura system was confirmed in a meta-analysis [12]. In addition, this scoring system has been found to be useful for predicting the DSS $(99.6 \%, 96.0 \%$, and $90.1 \%$, respectively, in 5 years).

Management of Elderly Patients with eCura C-2 ESD for EGC

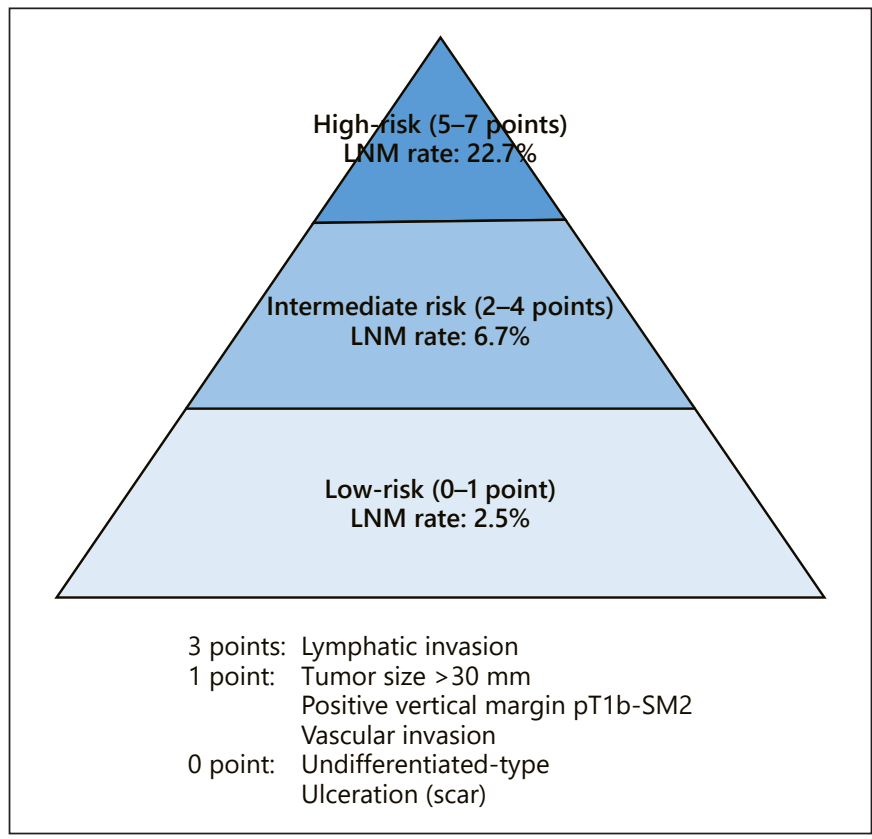

Fig. 2. eCura system. The eCura system, which consists of 5 pathological factors, is a risk scoring system that can predict the risk of LNM and gastric cancer-specific mortality after ESD with eCura C-2 for EGC. The LNM rates in the low-risk (0-1 points), intermediate-risk (2-4 points), and high-risk (5-7 points) categories were $2.5 \%, 6.7 \%$, and $22.7 \%$, respectively. LNM, lymph node metastasis; ESD, endoscopic submucosal dissection; EGC, early gastric cancer.

Then, is the eCura system perfect for predicting LNM after ESD with eCura C-2 for EGC? This system may not be perfect especially in undifferentiated-type EGC. Although 0 points were assigned to undifferentiated-type, this type has a potential for higher incidence of LNM than differentiated type even within the criteria for endoscopic resection [15]. The eCura system was established from the data after ESD; thus, many patients with undifferentiated-type EGC were not included in the development of the eCura system due to the limited indication of endoscopic resection for undifferentiated type [4]. Meanwhile, the application of the eCura system for undifferentiatedtype EGC after the current indication of endoscopic resection might be acceptable; however, due to a relatively small number of patients (292 patients) in the study [16], further validation with larger numbers of patients will be required.

Some findings about LNM after ESD for EGC were further shown. An undifferentiated component in submucosal invasion was one of the risk factors for LNM in submucosal cancer with eCura C-2 [17]. It was also re- 
ported that submucosal manipulation during ESD does not seem to enhance the risk for LNM or worsen the prognosis of the disease compared to gastrectomy [18].

\section{Metastatic Recurrence in Patients with No Additional} Treatment after ESD with eCura C-2 for EGC

Although the 5-year metastatic recurrence rate in patients with no additional treatment after ESD with eCura C-2 for EGC was 3.2\% [19], the rate differed across the risk categories of the eCura system. The 5-year metastatic recurrence rates in the low-, intermediate-, and highrisk categories were $0.7 \%, 5.7 \%$, and $11.7 \%$, respectively [13], and the risk of metastatic recurrence in patients with no additional treatment was significantly higher than that in those with additional gastrectomy only in the high-risk category [16]. However, unlike the cases with ESD for esophageal squamous cell carcinoma [20], it remains difficult for metastatic recurrence to be detected at a stage that can be curatively treated through salvage surgery in patients with no additional treatment after ESD with eCura C-2 for EGC. Indeed, only about $20 \%$ of the cases with metastatic recurrence after ESD with eCura C-2 and no additional treatment were detected as regional LNM in computed tomography during the follow-up [9], and few patients survived for long after the recurrence [21].

Furthermore, risk factors might differ with the time of recurrence. When metastatic recurrence was divided into early and late recurrence with the cutoff of 2 years, lymphatic invasion was a risk factor for early recurrence, whereas vascular invasion was that for late recurrence [19]. This fact might be useful in the management of elderly patients because a relatively short life expectancy is sometimes more important than a long one in elderly people.

\section{Metastatic Recurrence in Patients with Additional}

Gastrectomy after ESD with eCura C-2 for EGC

Metastatic recurrence in patients with additional gastrectomy after ESD with eCura C-2 for EGC is rare (1.3\% in 5 years) [22]. However, the status of LNM and vascular invasion might largely affect the rate of recurrence after additional gastrectomy [22]. Patients with N2/N3 in the TNM staging system had high rates of metastatic recurrence (27.3-42.9\% in 5 years), in contrast to the fact that the recurrence was scarcely shown in those with $\mathrm{N} 0(0.3-$ $0.5 \%$ in 5 years). In patients with $\mathrm{N} 1$, the risk of metastatic recurrence depends on vascular invasion: $19.4 \%$ and $0.0 \%$ in positive and negative vascular invasion, respectively, in 5 years.

\section{All-Cause Mortality}

\section{Current Knowledge of Predictors of All-Cause}

Mortality in Patients with ESD for EGC

Since chronological age alone is a poor descriptor of heterogeneity in the aging process, evidence-based methods of identifying the heterogeneity and predicting mortality are required. To date, several retrospective studies have evaluated the predictors of all-cause mortality after ESD for EGC. Although the Charson comorbidity index (CCI), Eastern Cooperative Oncology Group performance status, the American Society of Anesthesiologists' Physical Status, and prognostic nutritional index were reported as useful prognostic determinants, the results varied across the studies [23]. This might be because of the difference in candidate prognostic determinants among the studies and the presence of single- or 2-institution studies with a small number of included patients in the currently available literature. Furthermore, some important findings (e.g., cognition and psychological status) were not evaluated because of the retrospective study design.

Meanwhile, the association of risk categories of LNM such as eCura C-2 or additional treatment with all-cause mortality remains unclear. Although some studies showed no significant association between eCura C-2 and allcause mortality [24, 25], it might be difficult to make the definite conclusion because of the small sample size.

\section{Comprehensive Geriatric Assessment}

To evaluate the risk of all-cause mortality in elderly patients, comprehensive geriatric assessment (CGA) might be more appropriate. CGA is defined as a multidimensional, multidisciplinary process that identifies medical, social, and functional needs and the development of an integrated/coordinated care plan to meet those needs [26]. The main goal of CGA is to provide a comprehensive health appraisal to guide targeted geriatric interventions and appropriate cancer treatment selection [27]. While the domains of CGA in the currently available literature vary, the following domains are frequently used: (1) functional status, (2) comorbidity, (3) polypharmacy, (4) cognition, (5) psychological status (i.e., depression and anxiety), (6) social status and support, and (7) nutrition [26, 27]. Table 1 summarizes the domains and tools used in CGA.

Few studies have investigated CGA in patients with EGC. On the contrary, in the field of geriatric oncology, several recommendations were proposed from the American Society of Clinical Oncology (ASCO) for providing guidance regarding the practical assessment and management of vulnerabilities in elderly patients undergoing
Hatta/Gotoda/Koike/Uno/Asano/ Imatani/Masamune 


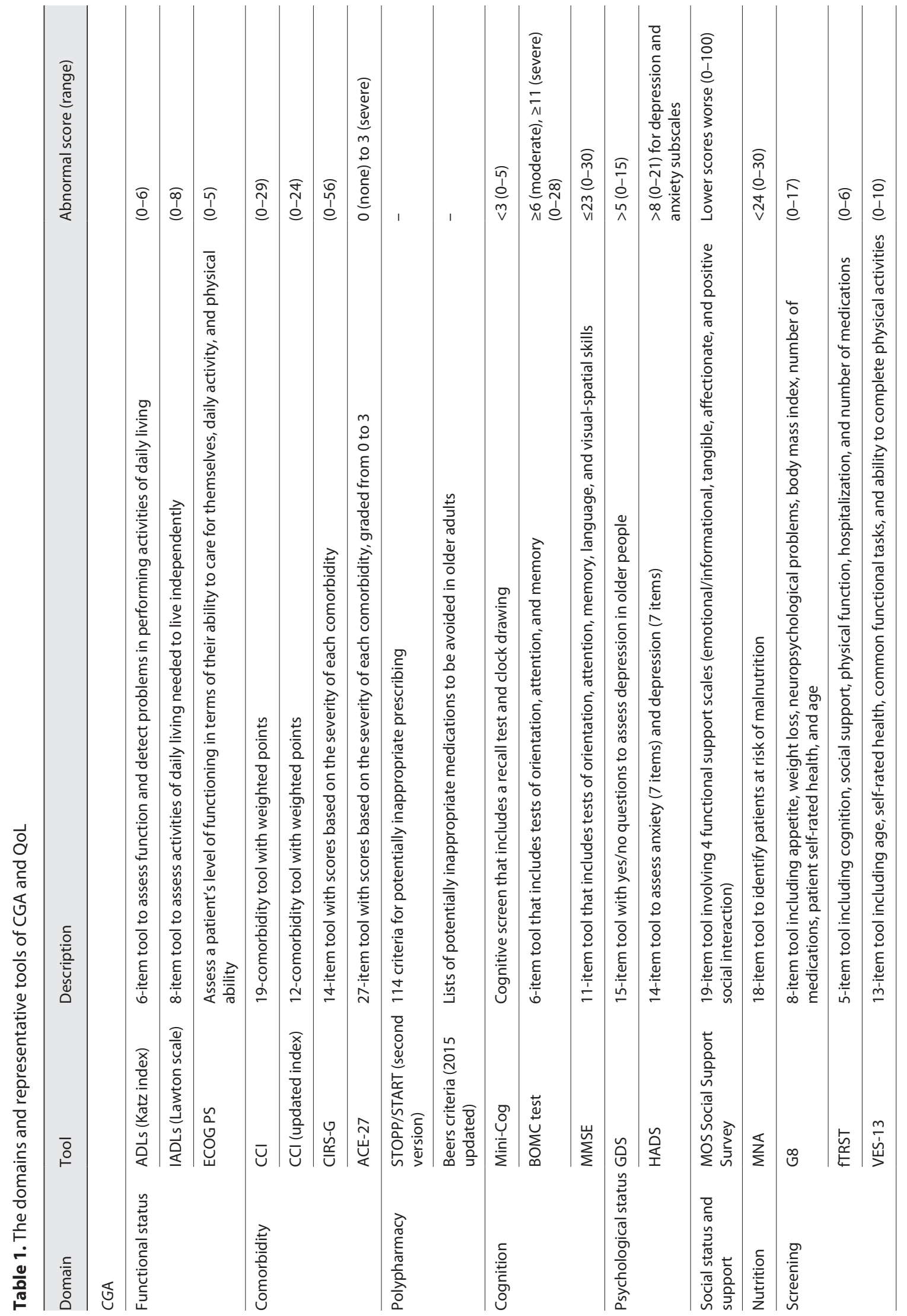


chemotherapy [28]. This guideline recommends several diagnostic tools: (1) instrumental activities of daily living to assess functional status, (2) a thorough history or a validated tool (such as the CCI) to assess comorbidity, (3) the singular question "How many falls have you had over the last 6 months (or since the last visit)"? on falls, (4) the Geriatric Depression Scale to screen for depression, (5) the Mini-Cog or the Blessed Orientation-Memory-Concentration test to screen for cognitive impairment, and (6) unintentional weight loss ( $>10 \%$ weight loss from the baseline weight) for nutrition [28]. However, one of the issues facing the use of CGA is that it is time consuming and difficult to implement in daily practice.

For short and easy administration, the use of geriatric screening tools has been gaining interest. The Geriatric- 8 and Flemish version of the Triage Risk Screening Tool have a strong prognostic value for functional decline and OS [29]. The Vulnerable Elders Survey-13 has also been considered a valuable discriminating tool for predicting functional decline or death [30].

\section{Quality of Life}

ASCO, the European Society of Medical Clinical Oncology, and the US Food and Drug Administration have recognized the QoL as a key secondary outcome criterion when evaluating anticancer treatment, especially when treatment interventions are not expected to alter patients' OS [5]. The integration of QoL and other patient-reported outcomes might become more frequent for supporting therapeutic decision-making.

A number of health-related QoL tools have already been reported (Table 1). Among them, the EORTC QLQ$\mathrm{C} 30$ [31] is one of the most widely used ones in cancer patients. This tool evaluates 3 main fields of the functional and clinical, psychological, and social environment; however, since this instrument was developed from the study including younger patients [31], this tool might not fully meet the needs of elderly patients [5].

The EORTC QLQ-ELD15 was developed to supplement the EORTC QLQ-C30 in elderly patients with cancer [32]. After the removal of 1 item (EORTC QLQELD15 $\rightarrow$ EORTC QLQ-ELD14), this questionnaire was internationally validated [33]. The short-form 36 and Functional Assessment of Cancer Therapy General also have some utility in elderly patients [5].

Some elements of QoL questionnaires, such as activities of daily living, look very similar to some parts of the CGA. However, QoL tools are designed to assess and identify not 


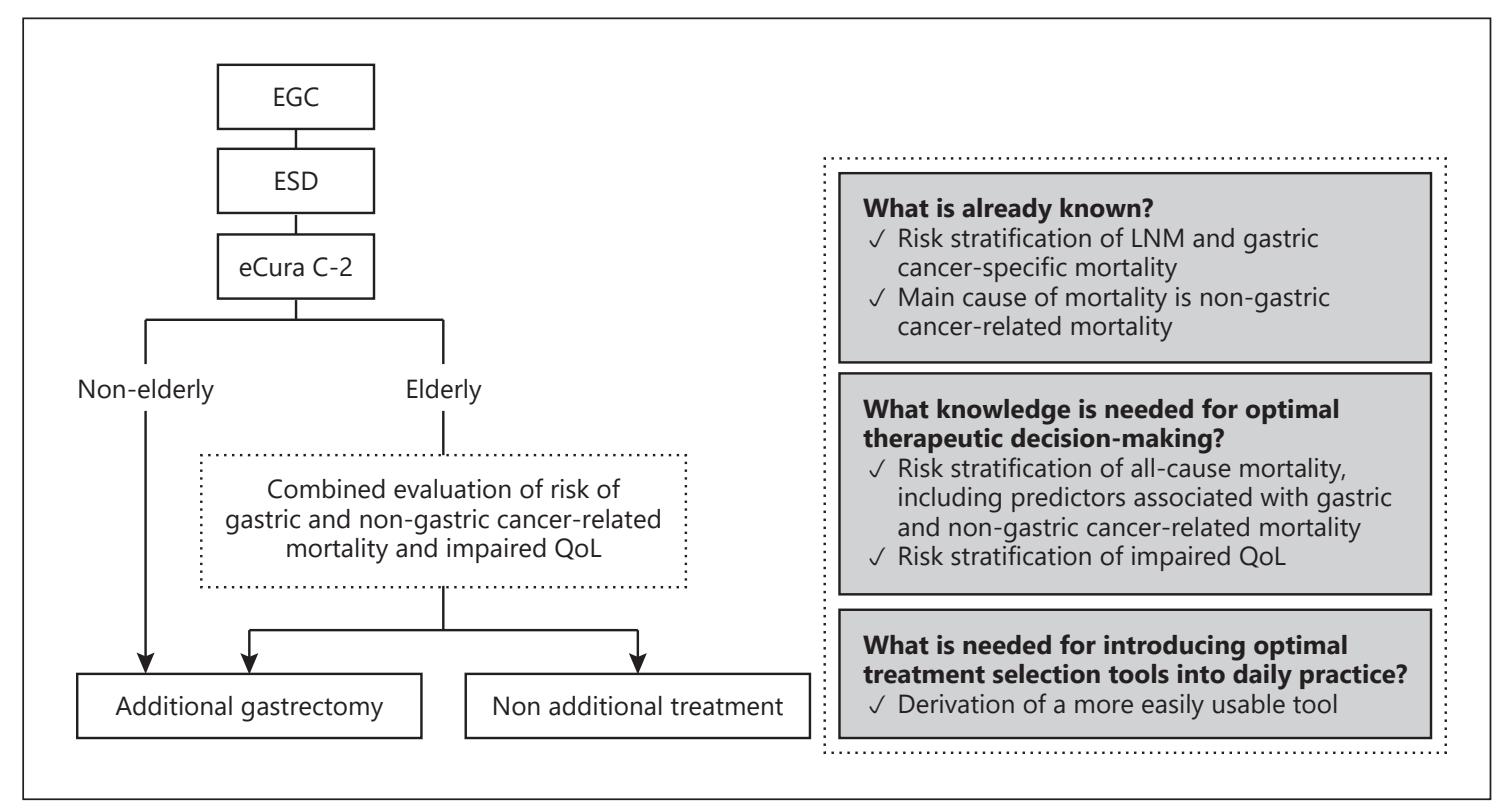

Fig. 3. Current knowledge and needs for optimal therapeutic decision-making in elderly patients after ESD with eCura C-2 for EGC. ESD, endoscopic submucosal dissection; EGC, early gastric cancer; QoL, quality of life; LNM, lymph node metastasis.

only what patients are capable of but also how the patients feel and what they believe about their capabilities [5].

In patients with EGC, some studies compared the impact of ESD or gastrectomy on QoL using EORTC QLQC30, EORTC QLQ-STO22 which is a gastric cancer-specific questionnaire, and so on $[34,35]$. However, the results are conflicting. According to Kim et al. [34], QoL parameters worsened the early posttreatment period in patients that underwent gastrectomy; however, most of them improved during the first posttreatment year, with differences between those with ESD and gastrectomy becoming insignificant. In contrast, Libanio et al. [35] showed a positive impact of endoscopic treatment on health-related QoL at 1 year after the treatment, without increasing the fear of recurrence and new lesions. However, the patients enrolled in these studies were not only elderly patients; thus, a comparison between treatment strategies in the elderly is required.

\section{Current Issues and Future Perspective for Optimal Management in Elderly Patients after ESD with eCura C-2 for EGC}

Although most available studies are retrospective, evidence of the risk of LNM and gastric cancer-specific mortality has been accumulated. However, since most patients after ESD with eCura C-2 for EGC die of nongastric cancerrelated causes, regardless of the treatment selection after ESD, all-cause mortality, including nongastric cancer-related mortality, is the most significant outcome. In addition, the QoL is also a significant outcome, as has been mentioned previously. A multimodal approach that includes CGA domains is required for identifying predictors of allcause mortality especially in elderly patients. Furthermore, it remains to be elucidated how much the risk categories of LNM, such as eCura C-2 with the high-risk category in the eCura system [13], and the selection of treatment methods affect all-cause mortality. To elucidate them, a large-scale nationwide multicenter prospective cohort study, known as the E-STAGE trial, is now ongoing in Japan (Trial No. UMIN000040910). We trust that this study will provide adequate information and evidence to enable the optimal management of elderly patients with EGC. Furthermore, one of the most important issues with CGA and QoL tools is that they are time-consuming. An easily usable tool for determining the prognosis and QoL should be developed and introduced into daily practice. Moreover, artificial intelligence has been recently applied in various medical fields. Its use might resolve these issues including how to combine the evaluation of 3 important outcomes (gastric cancer-specific mortality, nongastric cancer-related mortality, and QoL) to decide the treatment strategy after ESD, and the E-STAGE trial argues this important theme (Fig. 3). 


\section{Conclusions}

A combined evaluation of risk stratification of gastric cancer-specific mortality by the eCura system and the risk of nongastric cancer-related mortality and impaired QoL may be the current optimal method to decide treatment strategy after ESD with eCura C-2 for EGC in elderly patients. A large-scale prospective study that investigates CGA domains has been required to identify predictors of all-cause mortality and impaired QoL, and an easily usable tool should be developed.

\section{Funding Sources}

This study has not been funded.

\section{Author Contributions}

W.H. and T.G. contributed to conception and design; W.H. drafted the article; T.G., T.K., K.U., N.A., A.I., and A.M. contributed to critical revision of the article for important intellectual content; T.G. and A.M. contributed to study supervision.

\section{Conflict of Interest Statement}

The authors declare no conflicts of interest regarding this review article.

\section{References}

1 Mizota Y, Yamamoto S. How long should we continue gastric cancer screening? From an epidemiological point of view. Gastric Cancer. 2019 May;22(3):456-62.

2 Hatta W, Gotoda T, Koike T, Masamune A. History and future perspectives in Japanese guidelines for endoscopic resection of early gastric cancer. Dig Endosc. 2020 Jan;32(2): $180-90$.

3 Japanese Gastric Cancer Association. Japanese gastric cancer treatment guidelines 2018 (5th edition). Gastric Cancer. 2021 Jan;24(1): $1-21$.

4 Ono H, Yao K, Fujishiro M, Oda I, Uedo N, Nimura $\mathrm{S}$, et al. Guidelines for endoscopic submucosal dissection and endoscopic mucosal resection for early gastric cancer (second edition). Dig Endosc. 2021 Jan;33(1):4-20.

5 Scotte F, Bossi P, Carola E, Cudennec T, Dielenseger P, Gomes F, et al. Addressing the quality of life needs of older patients with cancer: a SIOG consensus paper and practical guide. Ann Oncol. 2018 Aug;29(8):1718-26.

6 Pilleron S, Sarfati D, Janssen-Heijnen M, Vignat J, Ferlay J, Bray F, et al. Global cancer incidence in older adults, 2012 and 2035: a population-based study. Int J Cancer. 2019 Jan; 144(1):49-58.

7 Esaki M, Hatta W, Shimosegawa T, Oyama T, Kawata N, Takahashi A, et al. Age affects clinical management after noncurative endoscopic submucosal dissection for early gastric cancer. Dig Dis. 2019;37(6):423-33.

8 Hatta W, Gotoda T, Koike T, Masamune A. A recent argument for the use of endoscopic submucosal dissection for early gastric cancers. Gut Liver. 2020 Jul;14(4):412-22.

9 Hatta W, Gotoda T, Oyama T, Kawata N, Takahashi A, Yoshifuku Y, et al. Is radical surgery necessary in all patients who do not meet the curative criteria for endoscopic submuco- sal dissection in early gastric cancer? A multicenter retrospective study in Japan. J Gastroenterol. 2017 Feb;52(2):175-84.

10 Suzuki S, Gotoda T, Hatta W, Oyama T, Kawata N, Takahashi A, et al. Survival benefit of additional surgery after non-curative endoscopic submucosal dissection for early gastric cancer: a propensity score matching analysis. Ann Surg Oncol. 2017 Oct;24(11):335360.

11 Dohi O, Hatta W, Gotoda T, Naito Y, Oyama $\mathrm{T}$, Kawata N, et al. Long-term outcomes after non-curative endoscopic submucosal dissection for early gastric cancer according to hospital volumes in Japan: a multicenter propensity-matched analysis. Surg Endosc. 2019 Dec;33(12):4078-88.

12 Hatta W, Gotoda T, Kanno T, Yuan Y, Koike T, Moayyedi P, et al. Prevalence and risk factors for lymph node metastasis after noncurative endoscopic resection for early gastric cancer: a systematic review and meta-analysis. J Gastroenterol. 2020 Aug;55(8):742-53.

13 Hatta W, Gotoda T, Oyama T, Kawata N, Takahashi A, Yoshifuku Y, et al. A scoring system to stratify curability after endoscopic submucosal dissection for early gastric cancer: "eCura system". Am J Gastroenterol. 2017 Jun;112(6):874-81.

14 Available from: https://apps.apple.com/app/ ecura/id1490245005 for iOS; https://play. google.com/store/apps/details?id=hatta.eCura for Android.

15 Abdelfatah MM, Barakat M, Lee H, Kim JJ, Uedo N, Grimm I, et al. The incidence of lymph node metastasis in early gastric cancer according to the expanded criteria in comparison with the absolute criteria of the Japanese gastric cancer association: a systematic review of the literature and meta-analysis. Gastrointest Endosc. 2018 Feb;87(2):338-47.
16 Hatta W, Gotoda T, Oyama T, Kawata N, Takahashi A, Yoshifuku Y, et al. Is the eCura system useful for selecting patients who require radical surgery after noncurative endoscopic submucosal dissection for early gastric cancer? A comparative study. Gastric Cancer. 2018 May;21(3):481-9.

17 Miyahara K, Hatta W, Nakagawa M, Oyama T, Kawata N, Takahashi A, et al. The role of an undifferentiated component in submucosal invasion and submucosal invasion depth after endoscopic submucosal dissection for early gastric cancer. Digestion. 2018;98(3):161-8.

18 Ito H, Gotoda T, Oyama T, Kawata N, Takahashi A, Yoshifuku Y, et al. Long-term oncological outcomes of submucosal manipulation during non-curative endoscopic submucosal dissection for submucosal invasive gastric cancer: a multicenter retrospective study in Japan. Surg Endosc. 2018 Jan;32(1):196-203.

19 Yamada S, Hatta W, Shimosegawa T, Takizawa K, Oyama T, Kawata N, et al. Different risk factors between early and late cancer recurrences in patients without additional surgery after noncurative endoscopic submucosal dissection for early gastric cancer. Gastrointest Endosc. 2019 May;89(5):950-60.

20 Hatta W, Koike T, Takahashi S, Shimada T, Hikichi T, Toya Y, et al. Risk of metastatic recurrence after endoscopic resection for esophageal squamous cell carcinoma invading into the muscularis mucosa or submucosa: a multicenter retrospective study. J Gastroenterol. 2021 Jul;56(7):620-32.

21 Takizawa K, Hatta W, Gotoda T, Kawata N, Nakagawa M, Takahashi A, et al. Recurrence patterns and outcomes of salvage surgery in cases of non-curative endoscopic submucosal dissection without additional radical surgery for early gastric cancer. Digestion. 2019;99(1): $52-8$.
90

Digestion 2022;103:83-91

DOI: $10.1159 / 000519514$
Hatta/Gotoda/Koike/Uno/Asano/ Imatani/Masamune 
22 Hatta W, Gotoda T, Oyama T, Kawata N, Takahashi A, Oka S, et al. Is additional surgery always sufficient for preventing recurrence after endoscopic submucosal dissection with curability C-2 for early gastric cancer? Ann Surg Oncol. 2019 Oct;26(11): 3636-43.

23 Hatta W, Gotoda T, Koike T, Masamune A. Management following endoscopic resection in elderly patients with early-stage upper gastrointestinal neoplasia. Dig Endosc. 2020 Sep; 32(6):861-73.

24 Sekiguchi M, Oda I, Suzuki H, Abe S, Nonaka $S$, Yoshinaga S, et al. Clinical outcomes and prognostic factors in gastric cancer patients aged $\geq 85$ years undergoing endoscopic submucosal dissection. Gastrointest Endosc. 2017 May;85(5):963-72.

25 Iwai N, Dohi O, Naito Y, Inada Y, Fukui A, Takayama S, et al. Impact of the Charlson comorbidity index and prognostic nutritional index on prognosis in patients with early gastric cancer after endoscopic submucosal dissection. Dig Endosc. 2018 Sep;30(5):616-23.

26 Parker SG, McCue P, Phelps K, McCleod A, Arora S, Nockels K, et al. What is comprehensive geriatric assessment (CGA)? An umbrella review. Age Ageing. 2018 Jan;47(1):149-55.
27 Wildiers H, Heeren P, Puts M, Topinkova E, Janssen-Heijnen ML, Extermann M, et al. International Society of Geriatric Oncology consensus on geriatric assessment in older patients with cancer. J Clin Oncol. 2014 Aug; 32(24):2595-603.

28 Mohile SG, Dale W, Somerfield MR, Schonberg MA, Boyd CM, Burhenn PS, et al. Practical assessment and management of vulnerabilities in older patients receiving chemotherapy: ASCO guideline for geriatric oncology. J Clin Oncol. 2018 Aug;36(22):2326-47.

29 Kenis C, Decoster L, Van Puyvelde K, De Grève J, Conings G, Milisen K, et al. Performance of two geriatric screening tools in older patients with cancer. J Clin Oncol. 2014 Jan; 32(1):19-26.

30 Biganzoli L, Mislang AR, Di Donato S, Becheri D, Biagioni C, Vitale S, et al. Screening for frailty in older patients with early-stage solid tumors: a prospective longitudinal evaluation of three different geriatric tools. J Gerontol A Biol Sci Med Sci. 2017 Jul;72(7):922-8.

31 Aaronson NK, Ahmedzai S, Bergman B, Bullinger M, Cull A, Duez NJ, et al. The European organization for research and treatment of cancer QLQ-C30: a quality-of-life instrument for use in international clinical trials in oncology. J Natl Cancer Inst. 1993 Mar;85(5):36576.
32 Johnson C, Fitzsimmons D, Gilbert J, Arrarras JI, Hammerlid E, Bredart A, et al. Development of the European organisation for research and treatment of cancer quality of life questionnaire module for older people with cancer: the EORTC QLQ-ELD15. Eur J Cancer. 2010 Aug;46(12):2242-52.

33 Wheelwright S, Darlington AS, Fitzsimmons D, Fayers P, Arraras JI, Bonnetain F, et al. International validation of the EORTC QLQELD14 questionnaire for assessment of health-related quality of life elderly patients with cancer. Br J Cancer. 2013 Aug;109(4): 852-8.

34 Kim YI, Kim YA, Kim CG, Ryu KW, Kim YW, Sim JA, et al. Serial intermediate-term quality of life comparison after endoscopic submucosal dissection versus surgery in early gastric cancer patients. Surg Endosc. 2018 Apr;32(4):2114-22.

35 Libanio D, Braga V, Ferraz S, Castro R, Lage J, Pita I, et al. Prospective comparative study of endoscopic submucosal dissection and gastrectomy for early neoplastic lesions including patients' perspectives. Endoscopy. 2019 Jan;51(1):30-9. 